\title{
La evaluación formativa: un análisis desde la perspectiva de los estudiantes
}

Juan Antonio Llorens-Molina ${ }^{a}$

${ }^{a}$ ETS de Ingeniería Agronómica y del Medio Natural - Grupo IEMA, Universidad Politècnica de València. Mail: juallom2@qim.upv.es

\begin{abstract}
Results from weekly online self-assessment activities are shown in this work. It has been developed over two consecutive years in an introductory organic chemistry course. These activities were also complemented by the availability of short video recording lessons (polimedia) related to key issues of subjectmatter content. The objectives of this work were mainly to know the students' opinions concerning the usefulness of these educational resources, as well as some aspects of their design and mode of use. Likewise, self-assessment practice is also related to students' performance in term final examination. The results showed a strongly positive perception of both resources although they also suggest modifying some aspects of their design and mode of use, such as their timing and duration of their availability.
\end{abstract}

Keywords: Self-assessment, feed-back, formative assessment, chemistry, Poliformat, multiple choice test.

\begin{abstract}
Resumen
En este trabajo se presentan los resultados de la aplicación de actividades de autoevaluación online semanales. Estas han sido desarrolladas a lo largo de dos años consecutivos en un curso introductorio de química orgánica. Dichas actividades vienen también complementadas por la disponibilidad de grabaciones en video de corta duración (polimedia) centradas en aspectos clave del contenido. Los objetivos de este estudio son principalmente conocer las opiniones de los estudiantes acerca de la utilidad de estos recursos, así como de algunos aspectos de su diseño y utilización. Asimismo, también se relaciona la práctica de la autoevaluación con el rendimiento en el examen de la asignatura. Los resultados muestran una percepción claramente positiva de estos recursos, aunque también aconsejan modificar algunos aspectos de su diseño y modo de utilización, tal como el momento en que se utilizan y el tiempo de disponibilidad.
\end{abstract}

Palabras clave: Autoevaluación, retroalimentación, evaluación formativa, química, Poliformat, prueba de opción múltiple. 


\section{Introducción}

La evaluación como instrumento de aprendizaje se concreta en múltiples modos de evaluación formativa basados en diferentes recursos, tanto presenciales como online. El impacto de esta práctica educativa en el rendimiento académico es un hecho ampliamente contrastado en múltiples investigaciones realizadas en contextos similares al de este trabajo: asignaturas básicas al comienzo de la educación superior (Parker y Loudon, 2012). Tal como indican Sadler y Good, (2006), la autoevaluación contribuye a la mejora del aprendizaje a través de cuatro clases de aportaciones: (1) pedagógicas, dado que la retroalimentación proporciona oportunidades para el cambio conceptual y la profundización en los contenidos, así como para adquirir nuevas habilidades y recursos metodológicos; (2) metacognitivas, al contribuir a una mayor consciencia por parte de estudiante acerca de sus carencias y limitaciones, potencialidades y evolución de su aprendizaje, (3) de carácter práctico, ya que si se le asigna también una función sumativa, supone un importante ahorro de tiempo para el profesorado, sobre todo cuando imparte docencia a grandes grupos y (4) afectivas, ya que el alumno percibe la evaluación como un instrumento de aprendizaje más que como un elemento meramente sancionador de sus logros académicos.

Considerando estas aportaciones, y pese a la evidencia de un impacto positivo en el rendimiento académico, puede afirmarse que la aplicación de la evaluación formativa no necesita justificarse por su eficacia, ya que es una consecuencia directa de una concepción del aprendizaje como un proceso continuo de reestructuración cognitiva en el que la actividad metacognitiva del estudiante cobra un protagonismo esencial. Tal como afirman Ibabe y Jauregizar, (2010) la práctica de la autoevaluación es un factor que contribuye en gran medida al desarrollo de las habilidades metacognitivas de los estudiantes, por lo cual favorece el desarrollo de un conocimiento crítico del estudiante acerca de su propio aprendizaje (Wiediger y Hutchinson, 2002).

Por este motivo, este trabajo no tiene como objetivo comparar el rendimiento académico de los estudiantes tras la aplicación o no de una estrategia de evaluación formativa. Desde una perspectiva ética no hay motivo para privar a un grupo de estudiantes de una práctica educativa cuya bondad está suficientemente fundamentada, tanto teórica como empíricamente tanto en lo relativo a la mejora del aprendizaje como a su percepción positiva por los estudiantes. (McDonald y Boud, 2003; Sadler y Good, 2006; Poulos y Mahony, 2008). Sin embargo, hay múltiples aspectos en el diseño de la evaluación formativa que son susceptibles de análisis y mejora, siendo para ello una dato especialmente valioso la opinión de los estudiantes. También cabe precisar que, a diferencia del enfoque que generalmente se asigna a la autoevaluación en la literatura, como complemento o alternativa a la evaluación sumativa, en este trabajo la evaluación formativa se plantea exclusivamente con un objetivo orientador y de mejora del aprendizaje. Consecuentemente, es necesario también describir el contexto en que las actividades de autoevaluación se utilizan, tanto en relación al diseño global del proceso de aprendizaje como al resto de actividades de evaluación. Estos aspectos serán tratados detalladamente al describir el desarrollo de la innovación.

(cc) EY-NC-ND 2017, Universitat Politècnica de València 


\section{Objetivos}

Los objetivos de este trabajo se centran en la aplicación de dos innovaciones concretas:

- La realización semanal de autoevaluaciones online, con feed-back inmediato a través de la herramienta "exámenes" de Poliformat.

- La introducción de grabaciones en polimedia destinadas a reforzar los contenidos del programa considerados a priori como más difíciles o relevantes, poniendo así, a disposición del estudiante una herramienta que le permite revisarlos en cualquier momento.

Esta última herramienta se plantea como un aspecto más de la evaluación formativa, al hacer posible una retroalimentación de mayor alcance que la proporcionada por la mera corrección de la prueba de opción múltiple. De este modo, los estudiantes pueden, a la vista de sus carencias, recurrir a las grabaciones para aclarar dudas y profundizar en los conceptos implicados.

Más concretamente, el objetivo de este trabajo es analizar desde la perspectiva de los estudiantes, los siguientes aspectos de las actividades de autoevaluación:

- Su utilidad en general, así como el grado en que facilitan llevar al día la asignatura

- Aspectos de su diseño como la extensión, periodicidad y número máximo de envíos.

- Modo de proporcionar la retroalimentación.

- Momento en que el estudiante la realiza.

En cuanto a las grabaciones en polimedia, se pretende conocer también:

- El grado en que son utilizadas y en qué momentos.

- Si se visualizan completamente.

- En qué medida es valorada su utilidad.

\section{Desarrollo de la innovación}

El presente trabajo ha sido realizado a lo largo de dos cursos consecutivos (2015-16 y 201617) en la asignatura Fundamentos Químicos para la Ciencia y Tecnología de Alimentos, en el primer curso de dicho grado. Esta materia consta de tres unidades didácticas de una duración aproximada de diez semanas cada una. En la primera de ellas, dedicada a la introducción a la química orgánica, se ha desarrollado la innovación descrita en este trabajo.

Desde el punto de vista metodológico, la unidad dedicada a la química orgánica está planteada como una transición a la "clase invertida". Sus contenidos están divididos en dos subunidades claramente definidas: una parte introductoria, dedicada a los conceptos básicos, y otra, en la que se tratan las diferentes familias de compuestos orgánicos en cuanto a estructura, formulación y nomenclatura, propiedades físicas y reactividad, con un 
enfoque claramente agroalimentario. Para la subunidad de conceptos básicos, el alumnado tiene disponible en "contenidos" de poliformaT un material didáctico estructurado a modo de curso online, de modo que puede seguir la asignatura de modo relativamente autónomo, estando las clases presenciales centradas preferentemente en la realización y discusión de ejercicios prácticos. El contenido de la segunda subunidad va desarrollándose a través de las clases presenciales, alternando la presentación teórica con la realización de ejercicios de aplicación.

En cuanto a la evaluación, se lleva a cabo en sus tres aspectos: inicial, formativo y sumativo. La evaluación inicial es una sesión de trabajo presencial basada en el video: "Algunas ideas importantes al empezar a estudiar química orgánica" (https://riunet.upv.es/handle/10251/51372). Esta actividad se basa en una selección muy estricta de los prerrequisitos imprescindibles para comenzar la asignatura.

La evaluación formativa se basa en el empleo de dos recursos:

- Autoevaluaciones online publicadas cada viernes a través de "exámenes" de Poliformat, basadas en los contenidos impartidos esa semana -también alguna pregunta de contenidos anteriores-. Estas pruebas son de opción múltiple, con una extensión entre 10 y 20 ítems, no se restringe el tiempo para su realización, se permiten dos envíos y están disponibles hasta después del examen. La calificación del segundo es registrada automáticamente y la valoración conjunta del resultado de todas estas pruebas se contabiliza aumentando hasta en un $10 \%$ la nota obtenida en los exámenes, siempre que ésta sea superior a 4.0. En cada uno de los cursos se han realizado un total de 10 autoevaluaciones, siendo su contenido el mismo, salvo pequeñas modificaciones.

- Autoevaluaciones realizadas en clase utilizando el teléfono móvil mediante la aplicación "socrative". Su utilización es puntual y encaminada a centrar la atención del alumnado en aspectos particularmente relevantes, a modo de repaso para aclarar dudas.

La evaluación sumativa se desarolla a través tres actos de evaluación:

- Un primer examen parcial cuyo objetivo prioritario es familiarizar al alumnado con el formato y criterios de corrección utilizados en la asignatura. Su contenido está referido a la primera subunidad y vale un $30 \%$. Al final de la unidad, tiene lugar el segundo parcial cuyo contenido es opcional: puede referirse a toda la unidad, si el resultado del primero no fue satisfactorio, o bien estar centrado exclusivamente en la segunda subunidad, en cuyo caso se valora un $70 \%$.

- Independientemente de los exámenes, se plantea un trabajo voluntario (cuyo realización ha sido prácticamente generalizada en los dos cursos) consistente en la

(c) EY-NC-ND 2017, Universitat Politècnica de València 
elaboración en grupos de tres estudiantes de un poster (curso 2015-16) o de un "screencast" (2016-17), centrado en moléculas orgánicas de importancia agroalimentaria. Su calificación se realiza mediante coevaluación por parte de todo el grupo y se valora aumentando hasta en un $15 \%$ la nota global de la unidad, siempre que los exámenes se haya obtenido más de 4.0

Los datos relacionados con los objetivos del trabajo han sido obtenidos a partir de un conjunto de cuestiones planteadas como ítems de opción múltiple o mediante la aplicación de escalas Likert, incluidos como anexo a la última prueba de autoevaluación semanal.

\section{Resultados}

De cierto interés pueden ser los resultados globales de las autoevaluaciones, así como su correlación con el rendimiento académico (nota global de los exámenes, sin contabilizar la realización de las propias autoevaluaciones y los trabajos en grupo) y la mayor o menor puntuación media alcanzada en cada una de las autoevaluaciones.

La tabla 1 refleja estos resultados, considerando separadamente cada curso. Se muestra el promedio de las autoevaluaciones y las puntuaciones máxima y mínima registradas. Se incluye también la calificación media del examen y el coeficiente de correlación lineal con su significación entre las puntuaciones medias de las autoevaluaciones y la del examen.

Tabla 1. Resultados de las pruebas de autoevaluación y de los exámenes

\begin{tabular}{|l|c|c|c|c|c|c|}
\hline \multicolumn{1}{|c|}{ Curso } & $\mathrm{N}$ & $\begin{array}{c}\text { media } \\
\text { autoeval. }\end{array}$ & Rango & $\begin{array}{c}\text { media } \\
\text { examen }\end{array}$ & $\mathrm{r}$ & Sign. $\mathrm{r}$ \\
\hline $2015-16$ & 91 & 7.5 & $9.1-6.2$ & 5.5 & 0.18 & $\alpha<0.05$ \\
\hline $2016-17$ & 81 & 7 & $9.3-5.5$ & 5.2 & 0.34 & $\alpha<0.005$ \\
\hline
\end{tabular}

$\mathrm{N}$ : número de estudiantes evaluados

$\mathrm{R}$ : coeficiente de correlación lineal de Pearson

A partir de la tabla de valores críticos de $\mathrm{r}$ (Meliá, 2017) se desprende que la correlación lineal es estadísticamente significativa en ambos casos $(\alpha<0.05$ en 2015-16 y $\alpha<0.005$ en 2016-17, para contraste unilateral -test de una cola-). A partir de estos resultados puede aceptarse cierto valor predictivo al resultado de la autoevaluación con respecto al rendimiento obtenido en el examen. Estos resultados pueden considerarse convergentes con los referidos por Ibabe y Jauregizar, (2010) utilizando también herramientas online. No obstante, dicho valores deben interpretarse con cierta reserva por la influencia de los datos procedentes del colectivo de estudiantes relativamente desvinculado de la asignatura que, obteniendo calificaciones muy bajas en el examen, dejaron de realizar varias autoevaluaciones que son puntuadas con 0 . 
Se muestran a continuación los resultados globales para los dos cursos (120 cuestionarios procesados) de la encuesta relativa a la valoración de las actividades de autoevaluación por los estudiantes y de algunos factores de su diseño.

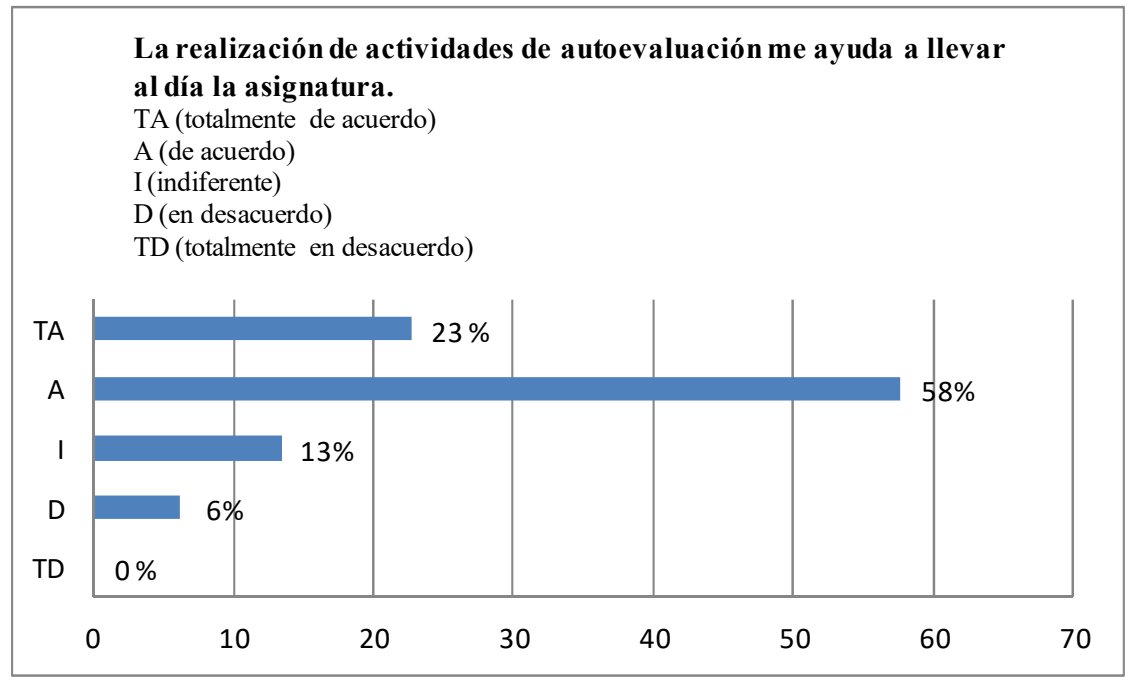

Fig. 1 Autoevaluación y seguimiento de la asignatura

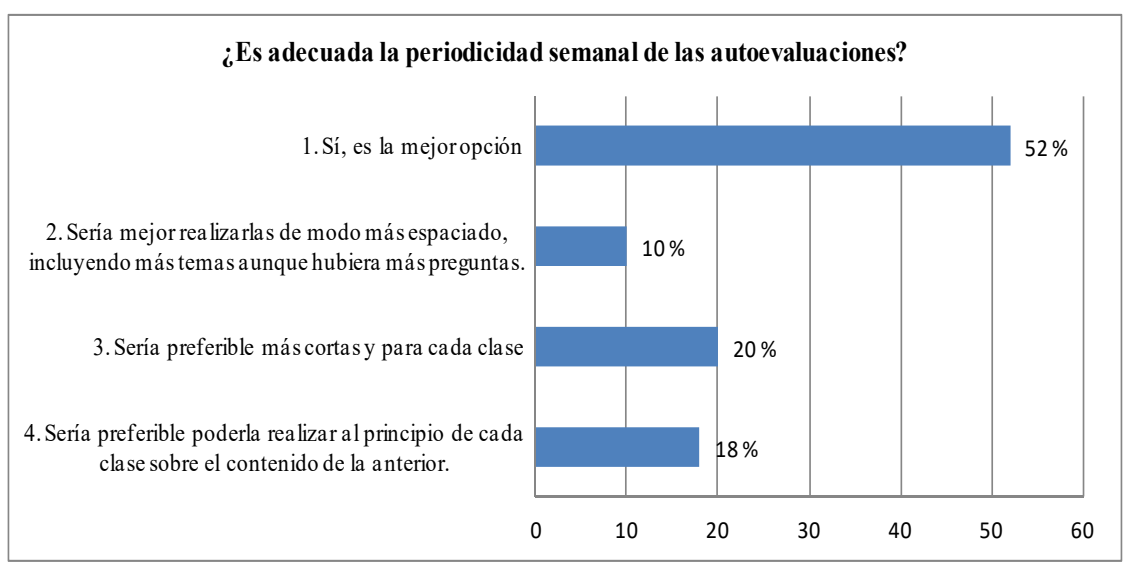

Fig. 2 Periodicidad de la autoevaluación

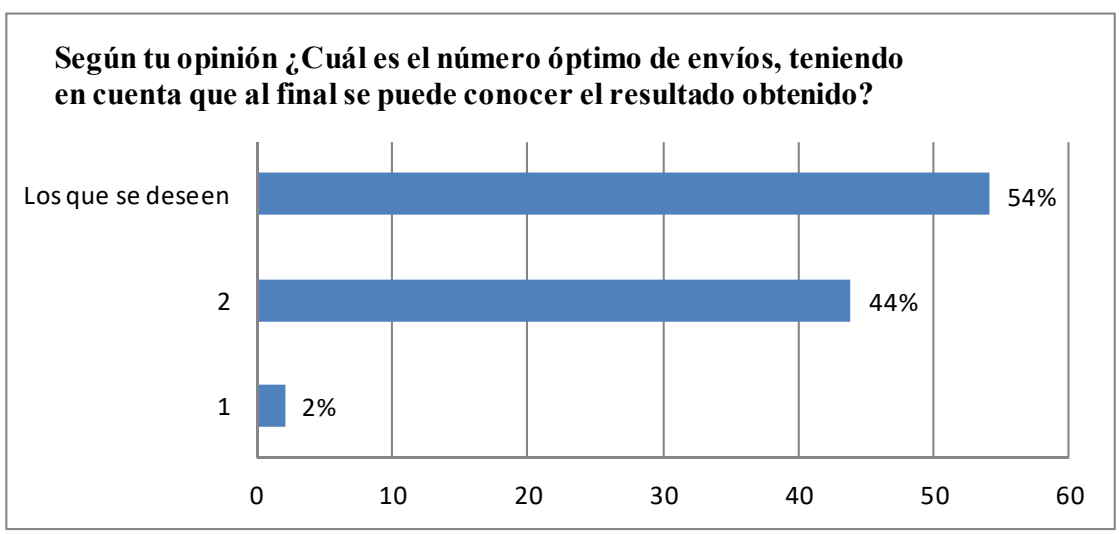

(cc) EY-NC-ND 2017, Universitat Politècnica de València 
Fig. 3 Preferencias acerca del número de envíos al realizar las pruebas de autoevaluación

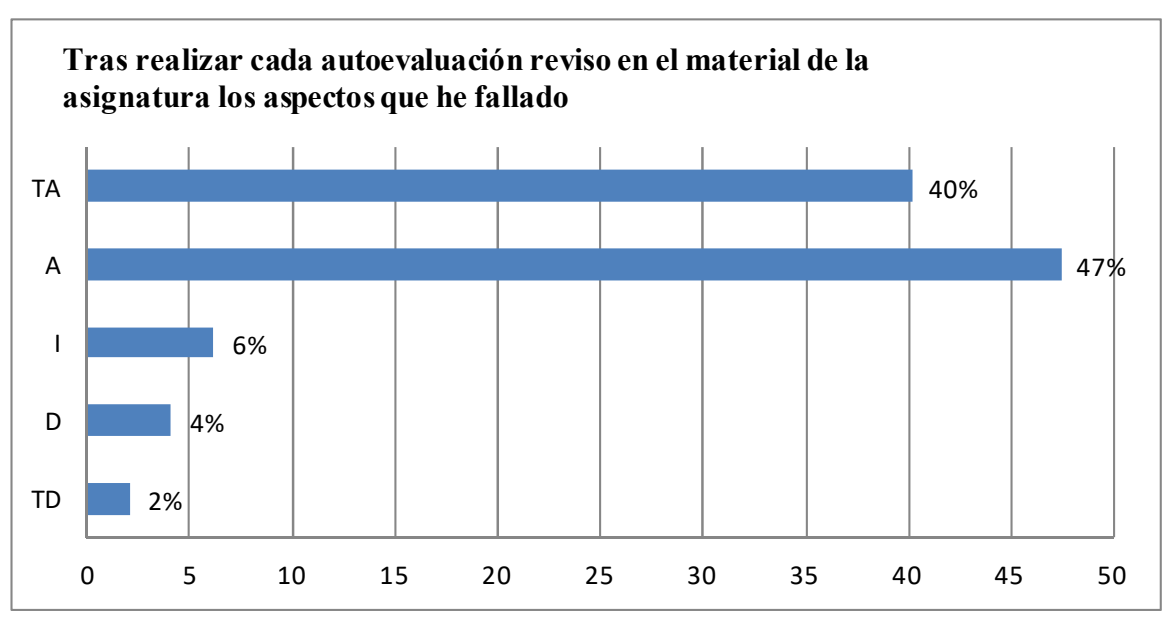

Fig. 4 Retroalimentación efectiva

¿Cuál es, a tu juicio, el número óptimo de preguntas para una autoevaluación semanal?

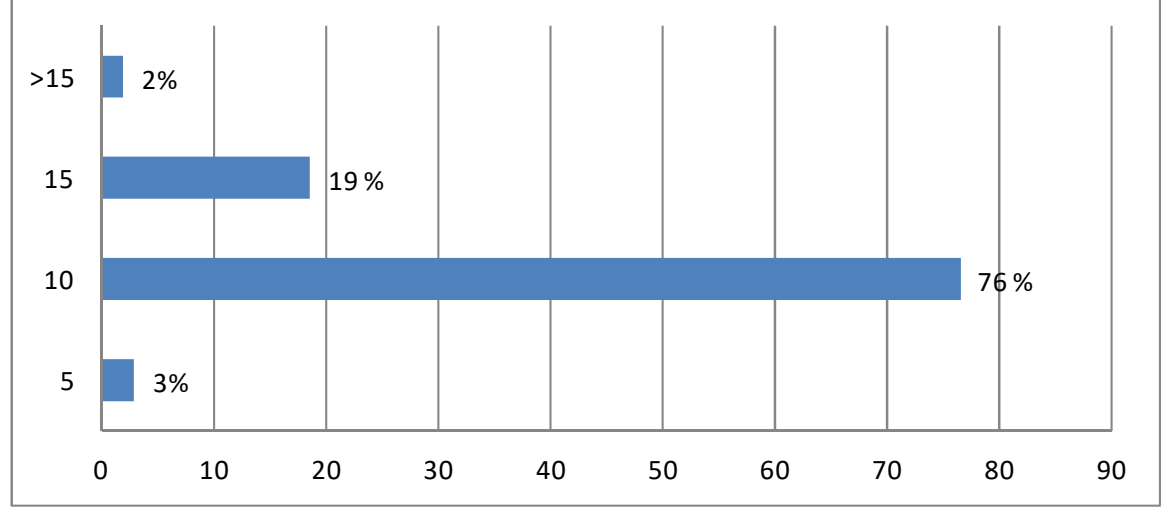

Fig. 5 Preferencias en cuanto a la extensión de las prebas

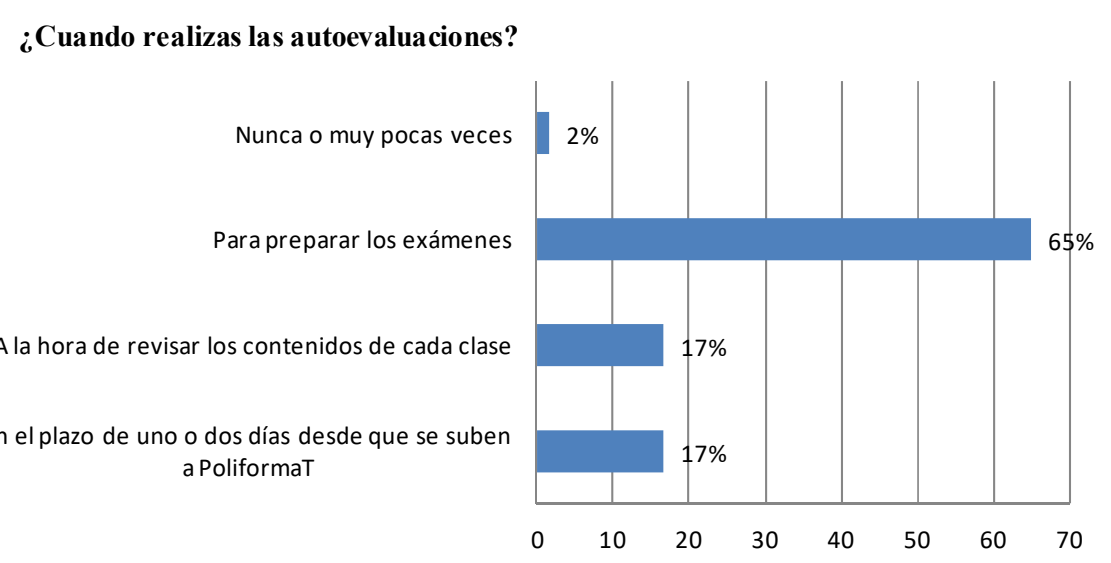

Fig. 6 Momento en que se realizan las pruebas de autoevaluación 


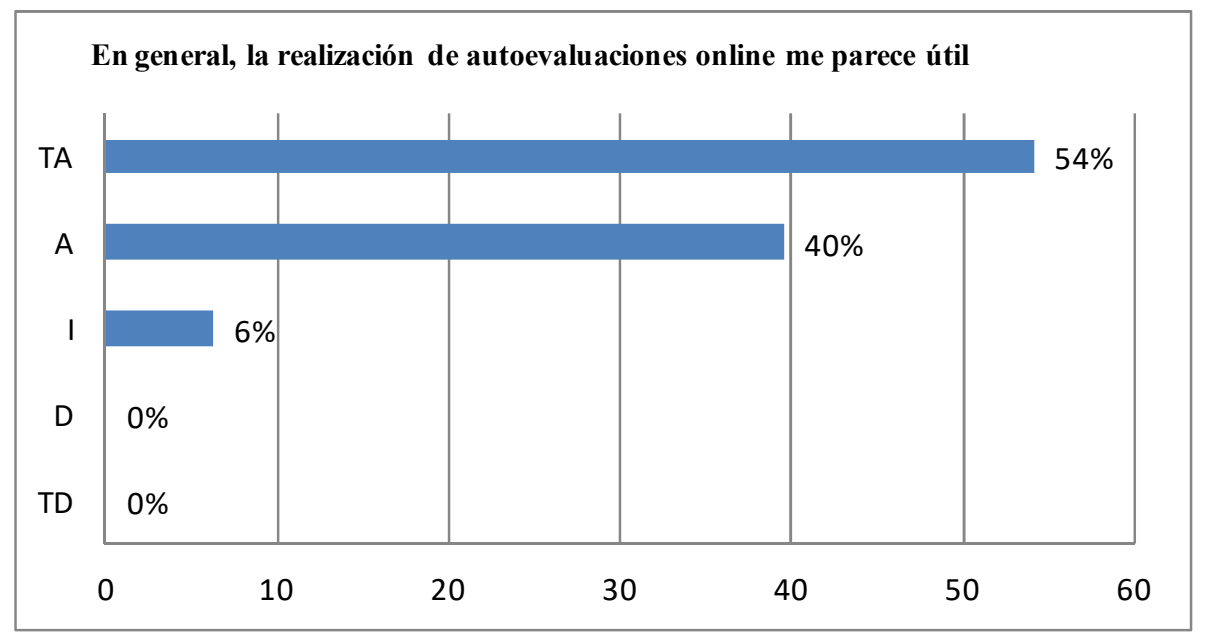

Fig. 7 Valoración general de la utilidad de la actividades de autoevaluación.

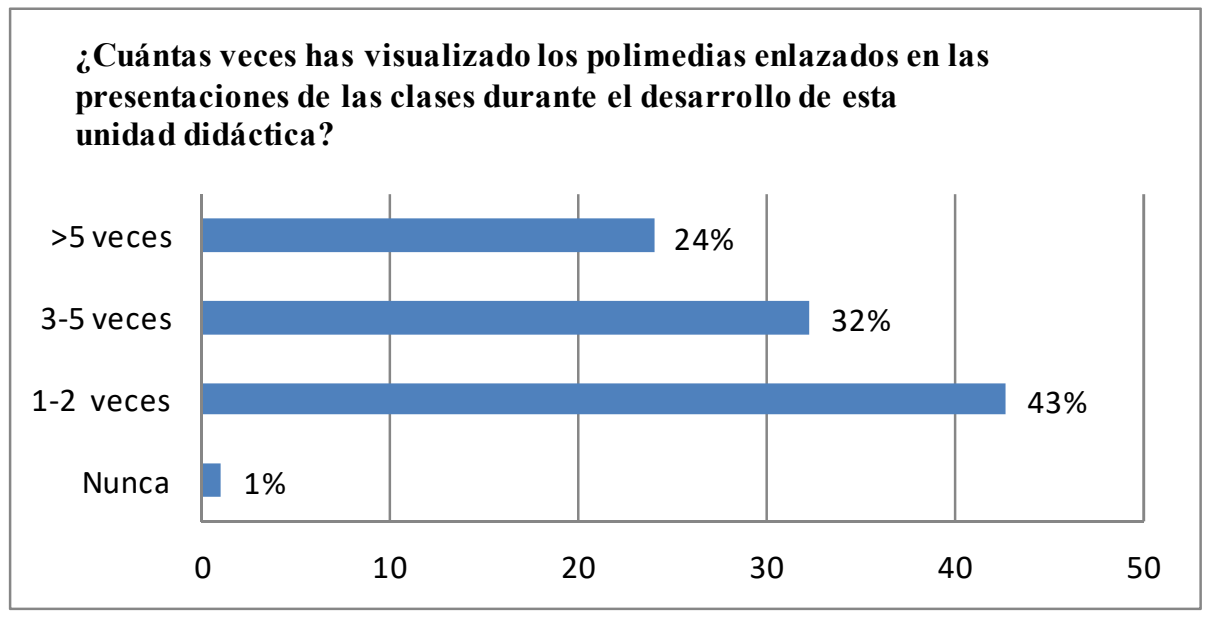

Fig. 8 Grado de visualización de las grabaciones polimedia

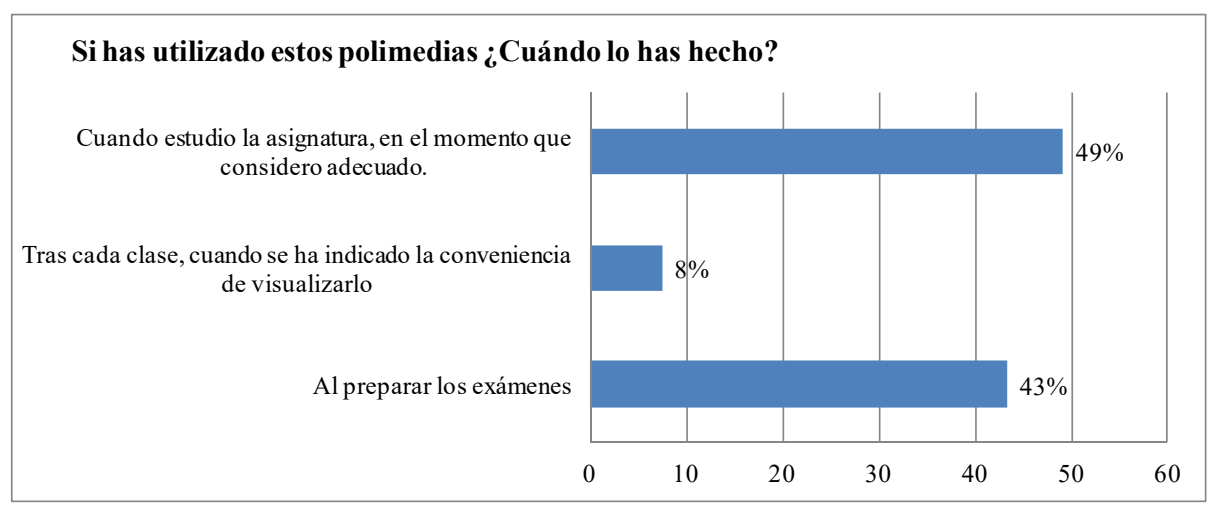

Fig. 9 Momento en que se utilizan las grabaciones polimedia 


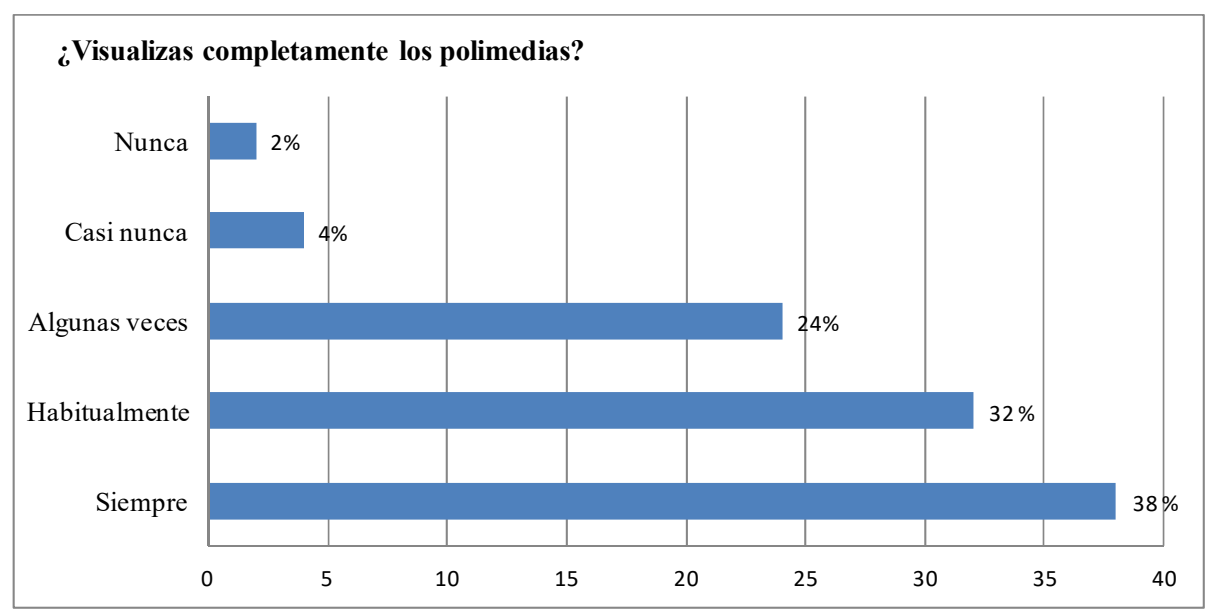

Fig. 10 Visualización completa de los polimedia

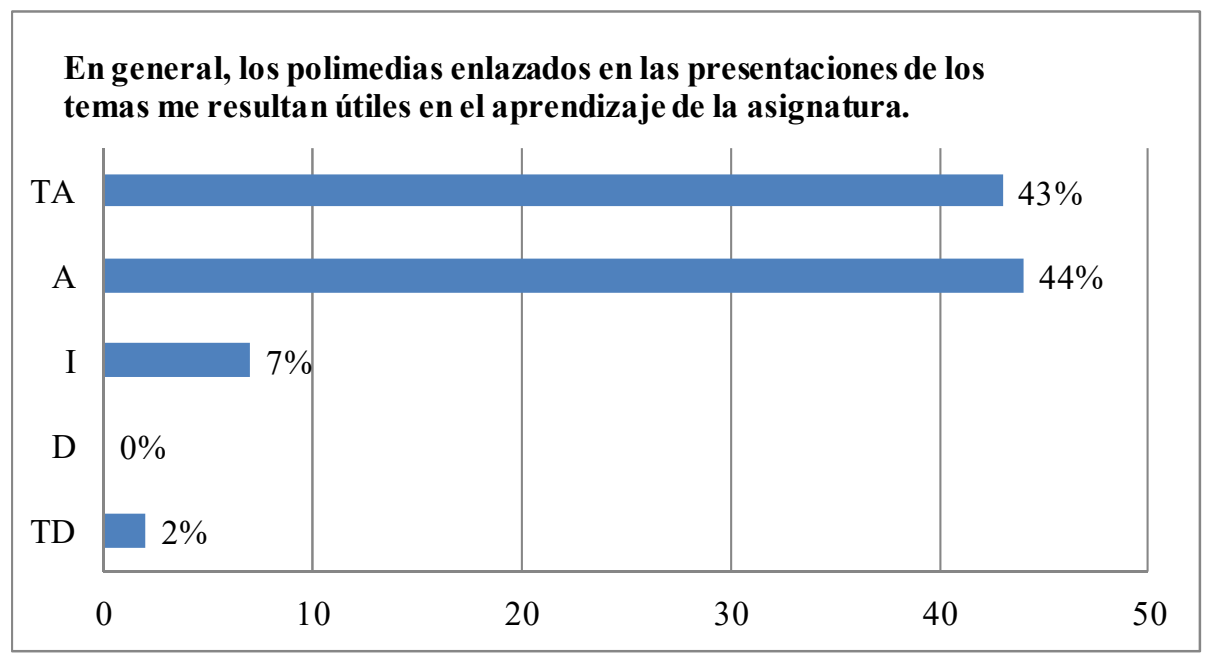

Fig. 11. Valoración general de la utilidad de las grabaciones polimedia

Los resultados muestran que la realización de actividades de autoevaluación es una práctica positivamente valorada por una gran mayoría del alumnado (Un $94 \%$ está de acuerdo o totalmente de acuerdo en su utilidad y un $81 \%$ también está de acuerdo o totalmente de acuerdo en que le ayuda a llevar al día la asignatura).

De estas valoraciones, consideradas globalmente, se desprende que los estudiantes perciben estos instrumentos como un complemento a su actividad académica cotidiana, más que como parte sustancial de la misma. Estos resultados son, en este sentido, coincidentes con los referidos por Ibabe y Jauregizar, (2010). Este carácter complentario es discutido por autores como McDonald y Boud, (2003), que proponen una mayor integración de la evaluación formativa en el diseño del curriculum como actividad capaz de modificar sustancialmente los ambientes de aprendizaje.

Acerca de su diseño y utilización, el alumnado manifiesta de modo mayoritario (87 \%) que la retroalimentación proporcionada al dar las soluciones correctas es acompañada por una 
revisión de los fallos con ayuda del material didáctico de la asignatura. La posibilidad de realizar un único envío es ampliamente rechazada siendo mayoritaria (54 \%) la opción por un número indefinido de envíos. Este hecho puede interpretarse teniendo en cuenta la seguridad que proporciona el obtener una calificación mayor tras recibir retroalimentación y repetir la actividad. Una vez más se confirma la importancia de la componente afectiva asociada a la evaluación formativa, a la que aluden diferentes autores como Poulos (2008), quién hace referencia explícita al efecto motivador e integrador en la vida universitaria que esto tipo de actividades aportan a los estudiantes de primer curso.

La periodicidad semanal es la opción más aceptada (52\%). No obstante, también es apreciable el número de estudiantes (38\%) que preferirían realizar la autoevaluación en cada clase, bien al principio (sobre la clase anterior), bien al final.

Existe un claro consenso ( $76 \%$ ) en torno al número más adecuado de ítems: 10. En cuanto al objetivo de su utilización, la preparación de los exámenes es la opción prioritaria (65\%), lo cuál puede explicarse teniendo en cuenta que son accesibles hasta el mismo momento del examen.

La introducción de grabaciones en polimedia como apoyo para la enseñanza presencial es valorada positivamente por una gran mayoría del alumnado (un $87 \%$ está de acuerdo o totalmente de acuerdo en su utilidad). Por otra parte, su grado de utilización es apreciable, aún teniendo en cuenta las reservas con qué deben interpretarse estos datos. Pese a que las estadísticas de visualización en upv media y youtube suelen mostrar aumentos puntuales del número de visualizaciones en las dos semanas previas a los exámenes, dado que éstas proceden también de ámbitos ajenos a la UPV, no es posible concluir de manera categórica que dichos aumentos son consecuencia de la preparación de los exámenes. Lo mismo puede comentarse acerca del grado en que los polimedia son visualizados completamente. Un $70 \%$ afirma que siempre o habitualmente proceden de este modo, pero la fiabilidad de este dato puede considerarse limitada.

En cuanto al momento de su utilización, un $92 \%$ de los estudiantes lo utiliza como material de repaso cuando estudian la asignatura y en la preparación de los exámenes. Su vinculación a cada clase presencial es muy limitada (8\%). Este hecho confirma la tendencia ya comentada anteriormente, acerca del carácter complementario que el alumnado asigna a estos recursos didácticos.

\section{Conclusiones}

Teniendo en cuenta las limitaciones que ofrece la interpretación de los datos de rendimiento académico en relación al desarrollo de las actividades de autoevaluación, existe una cierta evidencia de que lo favorecen. De todos modos, el contexto en que la actividad de autoevaluación se realiza es difícilmente controlable, por lo que cualquier predicción del rendimiento académico ha de realizarse con suma prudencia.

A partir de las preferencias del alumnado puede establecerse un perfil para las actividades de autoevaluación que podría definirse así:

(cc) EY-NC-ND 2017, Universitat Politècnica de València 
- Pruebas de 10 cuestiones planteadas al final de cada semana o bien al principio o final de cada clase (en este caso, su adaptación a socrative u otras aplicaciones similares utilizando el teléfono móvil sería la solución)

- Constituyen un recurso disponible en todo momento para el alumnado y lo que lo emplea discrecionalmente en función de su ritmo de trabajo, como un material didáctico más, a modo de complemento. No obstante, aún teniendo en cuenta estas preferencias, podría lograrse una mayor racionalización en el desarrollo de esta actividad abriéndola durante un tiempo limitado (una semana, por ejemplo) durante el periodo docente y volviéndolas a abrir durante un perido previo al examen para que sirviera como material de repaso.

- La retroalimentación consistente en proporcionar exclusivamente las respuestas correctas parace ser una opción adecuada, ya una elevada proporción de los estudiantes encuestados reconoce consultar el material docente de la asignatura para aclarar las dudas surgidas. El modo de aplicar la retroalimentación requeriría un estudio más específico y riguroso ya que puede ser la cuestión clave en el logro de una evaluación formativa eficaz.

Aún reconociendo la utilidad de las grabaciones en polimedia como recurso para ser utilizado discrecionalmente por el alumnado, un mayor control de su uso sería adecuado. En este sentido, cabría dirigir el diseño de los polimedia hacia la elaboración de verdaderas actividades de aprendizaje, dotándolas de interactividad. De este modo sería posible integrarlas como tareas específicas dentro del desarrollo ordinario de la asignatura. Se trataría, en definitiva, de elaborar un modelo de objeto de aprendizaje que, sin olvidar las características que lo definen, favorecieran una participación activa del alumnado cuando lo visualiza.

\section{Referencias}

IBABE, I. y JAUREGIZAR, J. (2010). "Online self-assessment with feedback and metacognitive knowledge”. Higher Education, vol. 59, 2, p. 243-258.

DOI 10.1007/s10734-009-9245-6 [consulta: 3 de marzo de 2017]

MCDONALD, B. y BOUD, D. (2003). "The impact of self-assessment on achievement: The effects of self-assessment training on performance in external examinations". Assessment in Education: Principles, Policy \& Practice, vol. 10, 2, p. 209-220.

MELIÁ, J. L. (2017). Valores críticos del coeficiente de correlación $r$ de Pearson. $<$ http://www.uv.es/meliajl/Docencia/Tablas/TablaR.PDF $>$ [consulta: 1 de marzo de 2017]

PARKER, L. L. y LOUDON, G. M. (2012). "Case study using online homework in undergraduate organic chemistry: results and student attitudes". Journal of Chemical Education, vol. 90, 1, p. 37-44.

POULOS, A. y MAHONY, M. J. (2008). "Effectiveness of feedback: The students' perspective". Assessment \& Evaluation in Higher Education, vol. 33, 2, p. 143-154.

SADLER, P. M. y GOOD, E. (2006). "The impact of self-and peer-grading on student learning". Educational assessment, vol. 11, 1, p. 1-31. 
$<\underline{\text { https://www.cs.auckland.ac.nz/courses/compsci747s2c/lectures/paul/2006_Sadler_PeerAssessment. }}$ pdf> [consulta: 6 de marzo de 2017]

WIEDIGER, S. D. y HUTCHINSON, J. S. (2002). "The significance of accurate student selfassessment in understanding of chemical concepts". Journal of Chemical Education, vol. 79, 1, p. 120.

DOI: 10.1021/ed079p120 [consulta: 3 de marzo de 2017] 